\title{
Las necesidades educativas especiales: un lastre conceptual para la inclusión educativa en España ${ }^{1}$
}

\author{
Alba García-Barrera a
}

\section{Resumen}

En España actualmente los maestros reciben una escasa formación sobre atención a la diversidad, y la titulación de Magisterio carece de la especialidad que antes preparaba a los orientadores escolares, cuya labor resulta esencial para el proceso de inclusión educativa. Además, el concepto de necesidades educativas especiales (NEE) parece estar suponiendo un lastre tanto para los propios alumnos a quienes hace referencia, como para los avances que tratan de hacerse para atender a la diversidad. A fin de conocer la opinión del sector educativo respecto a estas dos cuestiones, se realizó una encuesta a 160 profesionales en activo. Los resultados mostraron que los encuestados consideran clave el rol del orientador en este proceso, pero presentan una visión reduccionista de la diversidad. Por tanto, se considera que la formación tanto de maestros como de orientadores debería reforzarse a fin de hacer frente a esta problemática y que el concepto de NEE debería modificarse para evitar reduccionismos.

Palabras clave: Inclusión educativa. Necesidades educativas especiales. Educación especial. Orientación educativa.

\section{Introducción}

Desde los países escandinavos llegó, a mediados de los años 1970(WOLFENSBERGER, 1994) y tomando por bandera el principio de normalización, una corriente que supondría la base en la que se inscribirían los cambios que tendrían lugar a partir de 1978 con la difusión del Informe Warnock (WARNOCK, 1978). Dicho informe, realizado en Inglaterra, abarcó también los territorios de Escocia y Gales, y se convirtió en un fuerte punto de inflexión a la hora de percibir la educación especial y las personas que tradicionalmente han sido destinatarias de esta (UNESCO, 2005).

\footnotetext{
a Universidad a Distancia de Madrid. Madrid, España.

1 El presente artículo toma como base parte de la investigación realizada en la tesis doctoral presentada por la autora en la Universidad Autónoma de Madrid en el año 2013.
} 
En dicho Informe se sugirió por vez primera el término "necesidades educativas especiales", haciendo referencia a aquellos alumnos (uno de cada cinco según datos de la UNESCO, 2005) que precisan una o varias de las siguientes ayudas o apoyos a lo largo de su escolaridad (WARNOCK, 1978):

- Dotación de medios especiales de acceso al currículum a través de equipamientos especiales, provisión de recursos, modificación del ambiente físico o técnicas de enseñanza especializada.

- Provisión de un currículo especial o modificado.

- Particular atención a la estructura social y clima emocional donde tiene lugar la educación.

Así, y desde esta perspectiva, no son solo los niños con diversidad funcional quienes necesitan este tipo de ayudas, sino que hay otros tantos que, por diferentes causas, pueden llegar a requerirlas a lo largo de su escolaridad, de forma temporal o permanente, ya que las necesidades educativas especiales pueden variar en función de la evolución del alumno, del contexto en que se encuentre inmerso, y de la respuesta educativa que se le proporcione (UNESCO, 2005). Asimismo, esta noción nace con la idea de que el fin primero y último de la educación debe ser el mismo para todos los niños, independientemente de los problemas y dificultades a los que se enfrenten durante su desarrollo y su proceso de aprendizaje.

Dicho acontecimiento (el paso de un lenguaje centrado en las deficiencias a uno enfocado al concepto de Necesidades Educativas Especiales) ha supuesto uno de los cambios más importantes acontecidos en los últimos años en relación al tratamiento educativo de la diversidad, sumado al paradigma de inclusión educativa. En este sentido, se puede decir que la educación inclusiva tuvo su origen en el movimiento REI (Regular Education Iniciative), surgido en EE.UU. a mitad de los años 80 con base en la polémica suscitada con la reforma de enseñanza que se alentaba desde la Administración. Los principales objetivos de dicho movimiento consistían en convertir el sistema de educación especial y el de educación general en uno único, y en educar al mayor número posible de alumnos con diversidad funcional en las aulas ordinarias, de modo que pudieran compartir las mismas oportunidades y recursos de aprendizaje (GRAU RUBIO, 2001). Para ello resultaba indispensable cambiar las prácticas de los profesores y desarrollar nuevos programas educativos que fomentaran los sistemas consultivos y de enseñanza cooperativa, de modo que los maestros generalistas fuesen apoyados por los profesores especialistas en pos de una actuación conjunta dentro del aula 
ordinaria (REYNOLDS, 1989). Así, cobraba una enorme importancia el rol del orientador para lograr que los resultados de la inclusión en estos nuevos entornos fuesen los apropiados.

En razón a ello, la primera ley que estableció un modelo institucional no universitario de orientación educativa en España fue la Ley Orgánica 1/1990 (ESPAÑA, 1990) de Ordenación General del Sistema Educativo (LOGSE), considerando la atención psicopedagógica y la orientación educativa y profesional como uno de los 11 principios que deben guiar la actividad educativa (Art. $2.3 \mathrm{~g}$ ), llegando incluso a establecer la orientación educativa como factor clave de la calidad de la educación (Art. 55). Sin embargo, sus aspectos funcionales y organizativos no fueron regulados hasta la Ley Orgánica 9/1995 (ESPAÑA, 1995) de la Participación, la Evaluación y el Gobierno de los Centros (LOPEG), momento en que por fin las Administraciones educativas (central, autonómica y local) quedaron obligadas a garantizar la acción orientadora, según rezaba el artículo 60 de la misma.

Por todo ello, la labor de los orientadores y de los especialistas que llevaban a cabo dicho cometido supuso un punto primordial de apoyo para los maestros generalistas, convirtiéndose en piedra angular de las prácticas inclusivas.

Así, y ciertamente en los últimos 20 años, el mundo de la educación especial ha sido uno de los ámbitos que más ha contribuido, casi contradictoriamente, al avance hacia el modelo inclusivo. Sus profesionales realizaron grandes aportaciones a la transformación de los sistemas educativos ordinarios gracias a una visión más interactiva y contextual sobre los procesos de enseñanza-aprendizaje desprendida de su planteamiento sobre las necesidades educativas especiales (en adelante, NEE). También se ha debido a la presencia cada vez mayor de alumnos con NEE en los centros, que ha logrado hacer que estos se replanteen y rediseñen muchas de sus prácticas y proyectos educativos. Todo ello ha provocado una importante transformación en las actitudes sociales hacia la diversidad, que, sin embargo, no han dejado de aparecer junto con múltiples resistencias al cambio (ECHEÍTA, 2007), a las que, además, debe sumarse el constante devenir educativo que España sufre bajo la continua llegada de nuevas leyes y las modificaciones producidas en torno a la formación de los maestros.

Uno de los cambios que cabe destacar surgió a pesar de las grandes aportaciones por parte del sector de la educación especial y, más concretamente, de la labor de los orientadores educativos en el proceso inclusivo. Con la entrada del Espacio Europeo de Educación Superior en el año 2000, en España se comenzó a 
realizar una gradual adaptación de las titulaciones de Magisterio. A partir de ella, se prescindió de la especialidad de Pedagogía Terapéutica, principal encargada de la formación de estos profesionales. De este modo, se inició una marcada tendencia hacia la figura de un maestro generalista con unos conocimientos básicos sobre atención a la diversidad, que se contrapone a la necesaria e importante labor que los especialistas en orientación educativa estaban llevando a cabo dentro de los procesos inclusivos.

En aquellos momentos también se instauraba la Ley Orgánica 2/2006 (ESPANÃ, 2006) de Educación (en adelante, LOE), que planteaba una nueva terminología en relación a su ley predecesora: el concepto de Necesidades Específicas de Apoyo Educativo (en adelante, NEAE), que a su vez se ha mantenido en la recientemente aprobada Ley Orgánica 8/2013 (ESPAÑA, 2013) para la Mejora de la Calidad Educativa (en adelante, LOMCE). Así, se entiende por alumnos con NEAE aquellos que requieran una atención educativa diferente a la ordinaria por "necesidades educativas especiales, por dificultades específicas de aprendizaje, por sus altas capacidades intelectuales, por haberse incorporado tarde al sistema educativo, o por condiciones personales o de historia escolar", correspondiendo a las Administraciones educativas asegurar los recursos necesarios para que "puedan alcanzar el máximo desarrollo posible de sus capacidades personales y, en todo caso, los objetivos establecidos con carácter general para todo el alumnado" (ESPAÑA, 2006, Art. 71, ap. 2).

Por otra parte, se considera que los alumnos con NEE son aquellos que requieren "por un periodo de su escolarización o a lo largo de toda ella, determinados apoyos y atenciones educativas específicas derivadas de discapacidad o trastornos graves de conducta" (ESPAÑA, 2006, Art. 73). Sin embargo, resulta imprescindible subrayar en relación a ello el hecho de que "muchos niños -no solo los que sufren alguna discapacidad-experimentan dificultades de aprendizaje y tienen por lo tanto necesidades educativas especiales en algún momento de su escolarización" (PUJOLÀS, 2001: 25).

Sumado a estas limitaciones en la terminología empleada en la legislación educativa en España, cabe destacar la utilización de los sistemas de clasificación de la diversidad funcional, como el DSM-IV (penúltima edición del manual diagnóstico y estadístico de los trastornos mentales de la American Psychiatric Association - APA, 2000) o el CIE-10 (décima edición de la Clasificación Internacional de Enfermedades de la Organización Mundial de la Salud-OMS, 1992), que han producido a lo largo de los años un etiquetaje en el ámbito educativo con duros efectos negativos sobre el trato y el aprendizaje de los alumnos con dificultades. Se ha olvidado que dichos sistemas no dejan de ser un mero consenso en la forma de entender las diferencias individuales, y que únicamente sirven 
como marco para delimitar, en la medida de lo posible, dichas diferencias de cara a facilitar su posterior identificación, diagnóstico y tratamiento. Sin embargo, no siempre sus límites quedan claros ni su uso se reduce al estrictamente necesario. En ocasiones produce un encasillamiento nada útil a la hora de trabajar por la mejora en la calidad de vida de estas personas, tanto a nivel social (donde a veces se les señala o se resaltan características que no son propias de todos los individuos, estigmatizando al colectivo) como psicológico, educativo o sanitario.

En este sentido, Gallager (1976; citado en GARCÍA PASTOR, 2000) resaltó el efecto tranquilizador que puede llegar a tener el etiquetaje para algunos profesionales, ya que ofrece ciertas pautas para atender la problemática en cuestión que posee el alumno, obviando, sin embargo, los aspectos ecológicos que la rodean y las complejas condiciones sociales que se necesitarían para cambiarla.

Por su parte, Algozzine y Mercer (1980; citados en GARCÍA PASTOR, 2000) señalaron que el etiquetaje influye tanto sobre las percepciones y conducta del propio niño, como sobre las percepciones y conductas de las personas que interaccionan con este. De esta forma, sus efectos también generan expectativas y actuaciones personales determinadas que surgen a consecuencia de esta acción, así como ciertas percepciones y actuaciones de quienes le rodean. Y a su vez, la influencia que estas últimas poseen recae directamente sobre las expectativas y relaciones interpersonales que el sujeto etiquetado pueda tener (Ysseldyke y Algozzine, 1982; citados en GARCÍA PASTOR, 2000).

Además, dentro del contexto educativo identificar las dificultades de aprendizaje como "Necesidades Educativas Especiales" conlleva graves limitaciones. Supone una etiqueta que puede llevar a disminuir las expectativas y desviar la atención de las dificultades reales que está experimentando tanto el propio niño como otros que no tienen esta etiqueta, alejándonos de la fuente que origina el problema y que puede encontrarse "en las relaciones, las culturas, el tipo de actividades y recursos, el modo en que los profesionales apoyan el aprendizaje y el juego, y las políticas y la organización de los centros" (BOOTH et al, 2006, p. 5).

En este sentido, se considera que la labor del orientador resulta esencial a la hora de asesorar y apoyar al maestro en la supresión de aquellas barreras que impidan la adecuada participación y aprendizaje de estos estudiantes.

Sin embargo, dadas todas las contradicciones que han sido expuestas hasta el momento en el presente artículo acerca de la labor del orientador y del concepto de necesidades educativas especiales, se quiso averiguar si los profesionales del 
sector educativo español son conscientes del lastre que supone dicha noción, y si entre sus concepciones existen ciertos errores epistemológicos derivados de la terminología actualmente empleada. Además, se aprovechó el estudio para conocer la opinión que el profesorado manifiesta, por un lado, acerca de la importancia del orientador escolar para atender a la diversidad en las aulas, y por otro, acerca de la posición que mantiene en torno a la inclusión educativa.

\section{Método}

\subsection{Muestra}

La población de estudio estuvo formada por dos subgrupos:

- Expertos: entendidos como catedráticos y docentes de centros universitarios españoles, especialistas en el ámbito de la atención a la diversidad y la inclusión educativa.

- Profesionales: entendidos como gestores, directivos, orientadores y profesores de centros educativos públicos, concertados y/o privados correspondientes a enseñanzas no universitarias del ámbito educativo español.

En función de los resultados obtenidos en el cuestionario, se procedió a depurar la matriz de datos, eliminando todos los valores perdidos, tanto por los sujetos como por el propio sistema, y usando para ello el programa SPSS v17. Además, se tuvo en cuenta que:

- En las frecuencias absolutas de cada una de las categorías o valores que tomara la variable no existiera ningún valor ajeno a los valores lógicos de la variable.

- En el número de individuos de cada variable no se hubiera duplicado ningún sujeto.

- En el mínimo y el máximo (en la opción de estadísticos del cuadro de diálogo) no hubiera valores extremos que se distanciasen del rango lógico de la variable.

Después de depurar la matriz de datos, se procedió a efectuar un análisis exploratorio EDA (Exploratory Data Analysis), basado en el análisis descriptivo de cada variable. Para ello, se prestó especial atención a la detección de outliers o sujetos con puntuaciones que no llegasen o se alejasen notablemente de los modelos establecidos para el resto de individuos de la muestra. 
De esta forma, de la muestra inicialmente contactada (241 personas), se obtuvo la muestra final, compuesta por 160 sujetos.

a) Selección de la muestra

Parte del muestreo realizado fue no probabilístico, pero para enriquecer la muestra y tratar de que esta fuese más representativa, se conjugó con un muestreo probabilístico. En la Figura 1 se detallan los criterios de selección utilizados.

Finalmente, se hizo uso de un muestreo probabilístico, abriendo la encuesta a medios de difusión digitales, tales como Facebook, Twitter y LinkedIn, de modo que, partiendo de la red personal, el alcance se ampliaba a otros miembros secundarios e incluso externos a dicha red, ya que a su vez el enlace obtuvo una redifusión a través de distintos sujetos que tuvieron acceso al mismo por estos

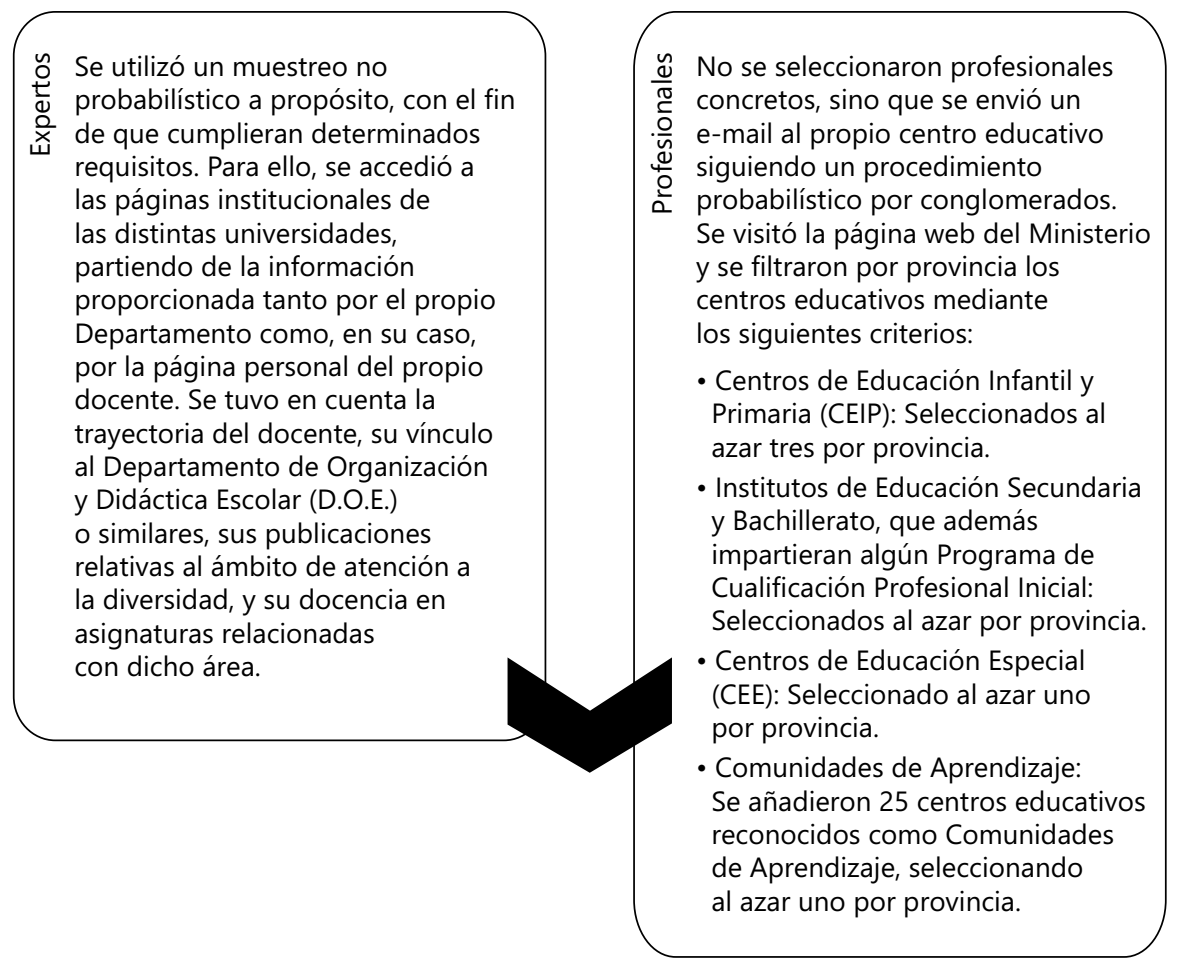

Fuente: Elaboración propia (2015).

Figura 1. Criterios de selección. 
medios. Por lo tanto, en ambas categorías (expertos y profesionales), existe cierto grado de muestreo probabilístico, lo que aumenta la representatividad de la muestra.

b) Variables

El cuestionario incluye, tal y como muestra la Tabla 1, variables de identificación y clasificación, tales como el correo electrónico del participante, su procedencia, su perfil (experto o profesional), su edad, su formación y su experiencia. Estos datos permiten categorizar y analizar los resultados en función de la respuesta ofrecida por cada sujeto.

c) Características de la muestra

Más de la mitad de los sujetos que componen la muestra final de estudio, concretamente 91 participantes (57\%), tienen una edad comprendida entre los 45 y los 65 años (Tabla 2).

Tabla 1. Variables del estudio.

$\leq 25$ años

Entre 26 y 35 años

Edad

Entre 36 y 45 años

Entre 46 y 65 años

$\geq 65$ años

Categoría

Profesionales

Expertos

$\leq 5$ años

Años de experiencia

Entre 6 y 10 años

Entre 11 y 19 años

$\geq 20$ años

Diplomatura

Nivel de formación

Licenciatura o grado

Estudios de postgrado

Título de doctor

Fuente: Elaboración propia (2015). 
Tabla 2. Edad de la muestra.

\begin{tabular}{lcc}
\hline Opciones de respuesta & Porcentaje de respuestas (\%) & Número de respuestas \\
\hline Menor de 25 años & 0 & 0 \\
Entre 26 y 35 años & 15,60 & 25 \\
Entre 36 y 45 años & 26,90 & 43 \\
Entre 46 y 65 años & 56,90 & 91 \\
Mayor de 65 años & 0,60 & 1 \\
\hline
\end{tabular}

Fuente: Elaboración propia (2015).

La mayoría de sujetos son profesionales (61\%), específicamente 97 de 160 sujetos, mientras que 63 son expertos (39,4\%). Gran parte $(47,5 \%)$ de la muestra de estudio cuenta con una experiencia profesional superior a 20 años (76 de los 160 participantes). $Y$ en cuanto a los estudios de los participantes, 53 participantes cuentan con un nivel de formación equivalente al título de Doctor (33\%), 47 han cursado una licenciatura o estudios de Grado (29\%), 32 poseen estudios de Postgrado (20\%), y 28 han realizado una diplomatura (18\%).

La mayor parte de los profesionales encuestados (88\%) trabaja en centros públicos, mientras que únicamente un $9 \%$ y un $3 \%$ trabaja en centros concertados y privados, respectivamente. El $40 \%$ de los participantes afirma trabajar en la etapa de Educación Primaria, el 37\% en Secundaria, y el 22,6\% restante se divide entre las etapas de Educación Infantil (11,3\%) y Bachillerato (11,3\%).

En cuanto a la categoría de expertos, la mayor parte (49\%) son profesores titulares universitarios, concretamente 31 de los 63 sujetos encuestados. Tras este perfil, los siguientes con una mayor representatividad en este estudio son el de catedrático y el de profesor asociado (12,7\% respectivamente), con 8 participantes cada uno, seguidos del perfil de profesor contratado Doctor ( $11,1 \%$ y 7 sujetos) y profesor colaborador $(6,3 \%$ y 4 sujetos).

\subsection{Instrumentos}

Se trata de una investigación no experimental basada en un estudio de encuesta de carácter descriptivo y corte transversal. La técnica de recogida de información consiste en un cuestionario realizado ad hoc puesto al alcance de los participantes a través de internet, concretamente mediante el servicio Survey Monkey. 
Durante el envío del cuestionario por correo electrónico, fue adjuntada una carta de difusión donde se presenta el concepto de Necesidades Educativas Personales a valorar por los participantes, descrito con un nivel de objetividad suficiente y sin inducir su opinión.

\subsection{Procedimiento}

Uno de los procedimientos que se ha seguido para asegurar la validez del cuestionario ha sido la aplicación de una prueba piloto y una validación por jueces.

Para medir la consistencia interna del cuestionario se ha llevado a cabo un Alfa de Cronbach, usando para ello el programa SPSS v17, obteniendo un resultado igual a 0,76 , por lo que se deduce que el cuestionario cuenta con una fiabilidad suficiente.

Como parte del análisis estadístico inferencial de los resultados, se ha llevado a cabo una prueba no paramétrica, concretamente $\chi^{2}$ (chi-cuadrado de Pearson), a fin de averiguar si existen diferencias significativas entre las distribuciones observadas y las teóricas o esperadas, y si algunos resultados de la investigación son extrapolables al resto de la población.

\section{Resultados}

La respuesta de los encuestados al ítem 1 (Tabla 3) muestra que el 96\% de los participantes conoce lo que se entiende según la LOE (ESPAÑA, 2006) por estudiantes con Necesidades Específicas de Apoyo Educativo, por lo que en

Tabla 3. Respuesta al ítem 1.

\begin{tabular}{|c|c|c|}
\hline Opciones de respuesta & $\begin{array}{l}\text { Porcentaje de } \\
\text { respuestas (\%) }\end{array}$ & $\begin{array}{l}\text { Número de } \\
\text { respuestas }\end{array}$ \\
\hline $\begin{array}{l}\text { Aquellos que presentan necesidades educativas } \\
\text { especiales. }\end{array}$ & 1,90 & 3 \\
\hline $\begin{array}{l}\text { Aquellos que presentan algún tipo de discapacidad y/o } \\
\text { dificultad. }\end{array}$ & 1,90 & 3 \\
\hline $\begin{array}{l}\text { Aquellos que presentan necesidades educativas } \\
\text { especiales, dificultades específicas de aprendizaje, altas } \\
\text { capacidades intelectuales, se hayan incorporado tarde } \\
\text { al sistema educativo, o tengan condiciones personales o } \\
\text { de historia escolar que requieran una atención educativa } \\
\text { diferente a la ordinaria. }\end{array}$ & 96,30 & 154 \\
\hline
\end{tabular}

Fuente: Elaboración propia (2015). 
este sentido se descarta un posible error cognoscitivo o conceptual a la hora de comprender a quiénes engloba dicho término.

En el ítem 2 se pregunta a los participantes por su concepción acerca de la noción de atención a la diversidad, entendiendo el $20 \%$ de ellos que este concepto contempla el hecho de proporcionar los recursos necesarios a los estudiantes con necesidades específicas de apoyo educativo (Tabla 4), considerando por tanto que son únicamente estos alumnos quienes verdaderamente representan la diversidad del aula.

El ítem 3 de la encuesta planteaba a los participantes el dilema de la localización, preguntándoles dónde consideraban que deberían ser atendidas las necesidades educativas de los alumnos que tienen mayores dificultades. El 79\% de los participantes, concretamente 126 personas de los 160 participantes, se mostraron a favor de que los alumnos con mayores dificultades estén en el aula ordinaria y sean atendidos tanto por el maestro generalista como por un especialista que desempeñe la figura de profesor de apoyo (ver Tabla 5), lo que parece suponer un avance en la aceptación del paradigma inclusivo por parte de los docentes.

Tabla 4. Respuesta al ítem 2.

\begin{tabular}{lcc}
\hline Opciones de respuesta & $\begin{array}{c}\text { Porcentaje de } \\
\text { respuestas (\%) }\end{array}$ & $\begin{array}{c}\text { Número de } \\
\text { respuestas }\end{array}$ \\
\hline $\begin{array}{l}\text { Lograr que todo el alumnado alcance el máximo } \\
\text { desarrollo personal, intelectual, social y emocional, así } \\
\begin{array}{l}\text { como los objetivos establecidos con carácter general en } \\
\text { la ley educativa vigente. }\end{array}\end{array}$ & 80,00 & 128 \\
$\begin{array}{l}\text { Proporcionar los recursos necesarios a los estudiantes } \\
\text { que tengan necesidades educativas especiales o } \\
\text { necesidades específicas de apoyo educativo. }\end{array}$ & 20,00 & 32 \\
\hline
\end{tabular}

Fuente: Elaboración propia (2015).

Tabla 5. Respuesta al ítem 3.

\begin{tabular}{lcc}
\hline Opciones de respuesta & $\begin{array}{c}\text { Porcentaje de } \\
\text { respuestas (\%) }\end{array}$ & $\begin{array}{c}\text { Número de } \\
\text { respuestas }\end{array}$ \\
\hline En un Centro de Educación Especial. & 5,6 & 9 \\
$\begin{array}{l}\text { Dentro del aula ordinaria, atendidos por un especialista } \\
\text { (profesor de apoyo). }\end{array}$ & 4,4 & 7 \\
$\begin{array}{l}\text { Dentro del aula ordinaria, atendidos por un especialista } \\
\text { (profesor de apoyo) y el maestro generalista. }\end{array}$ & 78,8 & 126 \\
\begin{tabular}{l} 
Fuera del aula ordinaria, atendidos por un especialista. \\
\hline
\end{tabular} & 11,3 & 18 \\
\hline
\end{tabular}

Fuente: Elaboración propia (2015). 
En contraste con el ítem 3, en el que el énfasis se encontraba sobre las dificultades de los estudiantes, el ítem 6 hace hincapié en la diversidad funcional de los alumnos, solicitando a los participantes que señalen aquella afirmación que más se aproxime a su opinión. El 68\% de los encuestados manifestó pensar que todo alumno, con independencia de sus capacidades y/o dificultades, debe ser atendido en el aula ordinaria con ayuda del maestro y de un profesor de apoyo, a diferencia de la respuesta ofrecida en el ítem 3, donde era el $79 \%$ de los sujetos el que se mostraba favorable a esta opción. Así, se observa una pérdida de un alto número de participantes que, al especificar la gravedad de las dificultades del alumnado, considera que deben ser atendidas fuera del aula ordinaria (ver Tabla 6).

Por otra parte, en el ítem 9 se indagaba acerca de la importancia que posee para los encuestados la figura del orientador en los centros educativos. Los resultados obtenidos y reflejados en la Tabla 7 muestran que un altísimo porcentaje de encuestados, concretamente el 98,1\%, considera esencial su labor.

En cuanto a la estadística inferencial llevada a cabo en el estudio, cabe destacar la relativa al ítem 2. En ella la hipótesis nula $\left(\mathrm{H}_{0}\right)$ fue refutada, ya que al aplicar

Tabla 6. Respuesta al ítem 6.

\begin{tabular}{lcc}
\hline Opciones de respuesta & $\begin{array}{c}\text { Porcentaje de } \\
\text { respuestas (\%) }\end{array}$ & $\begin{array}{c}\text { Número de } \\
\text { respuestas }\end{array}$ \\
\hline $\begin{array}{l}\text { Independientemente de sus capacidades y/o } \\
\text { dificultades, debe ser atendido en el aula ordinaria (con } \\
\text { ayuda del maestro y un profesor de apoyo). }\end{array}$ & 68,1 & 109 \\
$\begin{array}{l}\text { Que tenga diversidad funcional o serias dificultades de } \\
\text { aprendizaje, debe ser atendido fuera del aula ordinaria } \\
\text { (en clases de apoyo o refuerzo con ayuda de un }\end{array}$ & 25,0 & 40 \\
$\begin{array}{l}\text { especialista). } \\
\begin{array}{l}\text { Que tenga diversidad funcional o serias dificultades } \\
\text { de aprendizaje, debe acudir a un Centro de Educación }\end{array}\end{array}$ & 6,9 & 11 \\
Especial. & 6 & \\
\hline
\end{tabular}

Fuente: Elaboración propia (2015).

Tabla 7. Respuesta al ítem 9.

\begin{tabular}{lcc}
\hline Opciones de respuesta & Porcentaje de respuestas (\%) & Número de respuestas \\
\hline Sí & 98,1 & 157 \\
No & 1,9 & 3 \\
\hline
\end{tabular}

Fuente: Elaboración propia (2015). 
la prueba $\chi^{2}$ encontramos que el $\alpha$ obtenido es de 0,01 (ver Tabla 8) y que, por tanto, existen diferencias significativas para los rangos estudiados, tal y como se puede observar en la Tabla 9.

No obstante, las diferencias halladas en función del nivel de formación para este ítem únicamente son extrapolables al resto de la población para el cómputo global de la muestra estudiada (profesionales y expertos), no habiéndose detectado ninguna diferencia significativa en función de la formación al analizar por separado ambas categorías. Por tanto, puede inferirse al resto de la población una diferencia significativa en función de la formación adquirida a la hora de entender que el verdadero significado de atención a la diversidad consiste en lograr que todo el alumnado alcance el máximo desarrollo personal, intelectual, social y emocional, así como los objetivos establecidos con carácter general en la ley educativa vigente. Concretamente, y tal y como se aprecia en la Tabla 8, aquellas personas con una alta formación (título de Doctor) tienen muy claro, en su mayoría, que este concepto abarca a la totalidad de los alumnos, y que no consiste únicamente en proporcionar los recursos necesarios a los estudiantes con necesidades educativas especiales.

Tabla 8. Chi-cuadrado del ítem 2.

\begin{tabular}{cc}
\hline Variable & Sig. asintótica (bilateral) \\
Chi-cuadrado de Pearson & 0,017 \\
\hline
\end{tabular}

Fuente: Elaboración propia (2015).

Tabla 9. Diferencias del ítem 2 en función de la formación de los participantes.

\begin{tabular}{lccc}
\hline Formación & Sí & No & Total \\
\hline Diplomatura & 17 & 11 & 28 \\
Licenciatura o Grado & 39 & 9 & 48 \\
Postgrado & 25 & 7 & 32 \\
Título de Doctor & 47 & 5 & 52 \\
Total & 128 & 32 & 160 \\
\hline
\end{tabular}

Fuente: Elaboración propia (2015). 


\section{Conclusiones}

Las afirmaciones realizadas por los participantes acerca de la diversidad en los ítems 1 y 2 (Tablas 3 y 4) permiten concluir que, en España, el concepto de necesidades educativas especiales está suponiendo un lastre tanto para los propios alumnos a quienes hace referencia, como para los avances que tratan de hacerse en línea con la inclusión educativa, ya que, como puede apreciarse a través de sus respuestas, aunque los encuestados tienen claro lo que se entiende según la LOE (ESPAÑA, 2006) por estudiantes con necesidades específicas de apoyo educativo, un alto porcentaje de los mismos entiende por atender a la diversidad el hecho de proporcionar los recursos necesarios a estos estudiantes y no al resto. Esta visión reduccionista de la diversidad, enfocada únicamente a los alumnos que presentan una mayor problemática o carencia, responde a la idea de etiquetaje (AINSCOW, 1995; LÓPEZ MELERO, 1997; ALGOZZINE; MERCER, 1980; GALLAGER, 1976; YSSELDYKE; ALGOZZINE, 1982; citados en GARCÍA PASTOR, 2000) que se ha expuesto en este artículo. Se señala como diversidad lo que en lo ajeno se considera diferente, olvidando que la diferencia siempre es un concepto recíproco. Como trata de representarse mediante la Figura 2, aquello que se tilda de diferente en los demás es la misma diferencia que los demás pueden hallar en nosotros. Lo que siempre debe buscarse es ese camino interno que delimita los límites de las diferencias, marcado por los puntos en común que nos unen a los demás.

Todos somos diversos, todos somos diferentes y cada uno tiene sus propias Necesidades Educativas Personales (GARCÍA-BARRERA, 2013) a las que el sistema educativo debe dar, obligatoriamente, respuesta. Cada estudiante requiere que le sean proporcionados una serie de recursos y apoyos en función de sus necesidades, a fin de desarrollar al máximo su potencial individual.

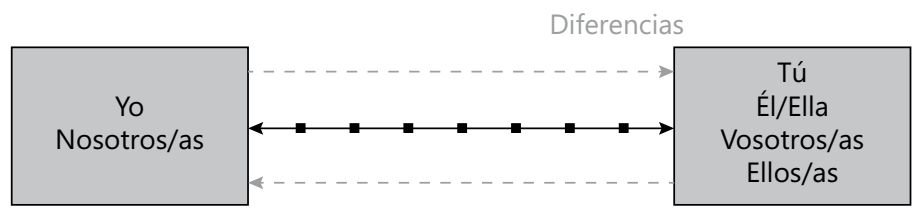

Puntos en común

El camino de las diferencias siempre lleva puntos en común

Fuente: Elaboración propia (2015).

Figura 2. El camino de las diferencias. 
La propia LOE (ESPAÑA, 2006) ya contemplaba como atención a la diversidad la respuesta indicada por el $80 \%$ de los encuestados en el ítem 2, lo cual se encuentra en completa sintonía con lo que se propone en el presente trabajo. No obstante, se considera que el concepto de Necesidades Educativas Personales podría contribuir a que ese $20 \%$ restante lograra entender que la diversidad somos todos.

En cuanto a las respuestas ofrecidas en el ítem 3 (Tabla 5), se aprecia cierto avance hacia la perspectiva inclusiva, ya que únicamente el $17 \%$ de los encuestados piensa que los alumnos con mayores dificultades deben estar en un Centro de Educación Especial (6\%) o ser atendidos fuera del aula ordinaria $(11 \%)$.

En este sentido, resulta necesario destacar que para atender adecuadamente las Necesidades Educativas Personales de todos los alumnos, incluyendo aquellos cuyas dificultades son más graves, debe diseñarse un aula abierta, organizada de forma flexible en función de las necesidades de cada estudiante, y, por supuesto, contar con la inestimable labor de especialistas y orientadores. Así, por ejemplo, si nos encontramos en la hora de Educación Física y tenemos un alumno con parálisis cerebral, este podrá ponerse a trabajar con el especialista y recibir la terapia física pertinente. Asimismo, en la hora de lengua y literatura podremos aprovechar para que realice su terapia miofuncional. El objetivo es que, por el hecho de que los alumnos tengan que estar juntos en un aula inclusiva, no caigamos nuevamente en la exclusión y queden olvidadas las Necesidades Educativas Personales de ciertos alumnos. Todos y cada uno de los estudiantes, sean cuales sean sus necesidades, deben ser igualmente atendidos. Debe construirse una Escuela Para Todos (Arnáiz, 2003) donde realmente cada estudiante reciba la atención educativa que necesita.

Para ello, se considera esencial la figura del orientador escolar, como muestran además los resultados obtenidos en el ítem 9. Pero en este sentido parece necesario recalcar que el peso y responsabilidad de la inclusión no debe recaer exclusivamente sobre los hombros de este profesional. Es esencial que los maestros generalistas posean amplios conocimientos sobre atención a la diversidad, dificultades de aprendizaje, diversidad funcional, síndromes e incluso psicopatologías, pero parece claro que no pueden ser expertos en todas y cada una de ellas. Debido a esta razón, contar con la ayuda y el asesoramiento de un orientador en los centros educativos se hace imprescindible (MARTÍNEZ-GARRIDO et al., 2010). Su colaboración, tanto dentro como fuera del marco del aula ordinaria para atender a las Necesidades Educativas 
Personales de todos los estudiantes, resulta de vital importancia para que las prácticas inclusivas no fracasen. En este sentido, tampoco debe olvidarse que para lograr este objetivo se deben facilitar los recursos personales necesarios a fin de ofrecer a cada estudiante la respuesta educativa que necesita. De este modo, se ha de poner a disposición del centro aquellos especialistas que requieran dichos estudiantes (LÓPEZ TORRIJO, 2009), así como las actuaciones, estrategias y recursos de atención a la diversidad necesarios, debiendo todo ello encontrarse a disposición de cada centro educativo de forma independiente al número de alumnos con dificultades que tenga matriculado.

Por otra parte, y retomando la diferencia apreciada entre las respuestas a los ítems 3 y 6 , cabe destacar que su origen parece residir en que en este último se hace referencia de una forma más concreta a la atención del alumnado con "diversidad funcional". De este modo, se comprueba cómo una mera etiqueta tiene el poder de cambiar la opinión de las personas. Así, parte de la muestra de estudio manifestó por medio de sus respuestas la convicción de que estos alumnos son "distintos" a los que presentan "mayores dificultades" (ítem 3), y que por tanto deben atenderse fuera del aula ordinaria.

Así, en el ítem 3 se detecta cierto avance hacia la visión inclusiva de la educación, ya que la mayor parte de los sujetos encuestados coincide en afirmar que los alumnos con mayores dificultades tienen que estar presentes en el aula ordinaria al igual que el resto de sus compañeros, debiendo ser atendidos dentro de esta, tanto por el maestro generalista como por un especialista que desempeñe la figura de profesor de apoyo. Sin embargo, cuando en el ítem 6 se hace hincapié en si los alumnos con diversidad funcional o graves dificultades de aprendizaje deben estar igualmente presentes en el aula ordinaria y ser atendidos, tanto por el maestro generalista como por un profesor de apoyo especialista, algunos participantes se retraen de la postura manifestada anteriormente, pasando así de un $79 \%$ de encuestados que apoyaban dicha cuestión, a solo un $68 \%$. Por tanto, queda de manifiesto nuevamente la visión reduccionista de la diversidad (Ainscow, 1995; Arnáiz, 2003) mantenida por algunos de los sujetos encuestados, así como la permanencia del dilema de la localización.

Aunque sigue siendo una mayoría de participantes quienes demuestran estar a favor de la inclusión de los alumnos con dificultades en el aula ordinaria, vemos cómo la respuesta varía en función de la supuesta gravedad de las mismas. Puede que esta disyuntiva se deba a la capacidad y/o preparación del propio profesorado para responder a las demandas educativas de estos alumnos (DURÁN GISBERT; GINÉ GINÉ, 2011; LEÓN GUERRERO, 1999; PALOMARES RUÍZ, 1998; 
PARRILLA, 2003), e incluso que tenga relación con los recursos disponibles en los centros para atenderlas (AINSCOW, 1999; DUK; LOREN, 2010).

De igual modo, por medio de las respuestas ofrecidas a dichos ítems se puede afirmar que aún hoy persiste el denominado dilema de las diferencias individuales (DYSON, 2001; NORWICH, 2008; LÓPEZ et al., 2010), relacionado con la valoración que se realiza con respecto a la diversidad humana (diferencias de capacidad, procedencia, identidad cultural, clase social, etc.) y con el intento de ofrecer a todo el alumnado tanto una igualdad de oportunidades efectiva en su aprendizaje y en su participación escolar, como una educación de calidad dentro de un marco común, inclusivo y comprensivo (ITE, 2009). Así, cabe señalar que los sistemas educativos se encuentran sujetos a una doble y contradictoria exigencia, basada en la necesidad de ofrecer una educación común para todos los alumnos $\mathrm{y}$, al mismo tiempo, reconocer sus necesidades individuales de aprendizaje y ser capaces de ajustarse a ellas de una forma apropiada (LÓPEZ et al., 2010).

Partiendo de la base de que todos, absolutamente todos, tenemos necesidades - de todo tipo; ya lo sugería Maslow (1943)-, es evidente que también todos tenemos necesidades educativas. Y dichas necesidades educativas deben ser cubiertas siempre con la máxima atención, a fin de desarrollar todo el potencial individual de cada persona. Es por ello que en este artículo se reivindica cambiar el término de Necesidades Educativas Especiales (NEE) por el de Necesidades Educativas Personales (NEP), ya que se considera que ello permitiría complementar el paradigma educativo inclusivo y fomentar la normalización de los estudiantes con dificultades, pasando de resaltar sus limitaciones a destacar sus capacidades y diseñar la práctica educativa en función de ellas. Para ello, como no podía ser de otra forma tras todo lo expuesto, se desea poner de relieve la imprescindible labor que los especialistas y orientadores escolares se ocupan de realizar en todo este proceso.

En definitiva, el enfoque reduccionista de la diversidad, en el que se pensaba en "alumnos distintos, con necesidades distintas que requieren respuestas distintas" (ARNÁIZ, 2003, p. 147), que agravaba los procesos de exclusión y limitaba las oportunidades de aprendizaje, ha de ser superado. La diferencia debe entenderse como un valor educativo enriquecedor para los procesos de enseñanza-aprendizaje llevados a cabo, tanto dentro del aula como fuera de ella. Pensar desde el punto de vista señalado no ha de ser necesariamente negativo si se contempla desde una perspectiva inclusiva, en el que estas diferencias sean tomadas en consideración a la hora de trabajar sobre ellas y sacarles partido en el aula, en lugar de interpretarlas como un elemento limitador y segregador promotor de desigualdades. 
El concepto de Necesidades Educativas Personales trata de aportar a ello desde un enfoque que considera que todos los alumnos son especiales y tienen unas necesidades educativas particulares, distintas de las de sus compañeros, que deben ser siempre atendidas y tomadas en consideración en el aula. De este modo, toma como verdadero centro de la enseñanza al propio estudiante en tanto persona única y distinta de las demás. Deja de poner el foco en las dificultades de los alumnos y se centra en sus capacidades, en el potencial que puede llegar a desarrollarse en cada uno de ellos. No diferencia ni segrega al alumnado en función de sus limitaciones, porque entiende que cada cual tiene unas, distintas además en cada momento de aprendizaje, y que el profesorado debe trabajar sobre ellas sin considerarlas un lastre para el aula ni basarse en ellas para etiquetar a los estudiantes. Por tanto, el concepto que se propone no consiste únicamente en un cambio terminológico, sino que conlleva toda una transformación de las prácticas educativas y de la visión que parte del profesorado tiene sobre la diversidad, pudiendo encontrarse una guía de buenas prácticas en este sentido en el trabajo de García-Barrera (2013), que el lector puede consultar para una mayor profundización en lo aquí expuesto.

Dichas prácticas inclusivas, cuyo fin último es mejorar el aprendizaje y el desarrollo del potencial individual y personal de cada estudiante, implican un importante cambio en la labor que maestros generalistas, especialistas y orientadores realizan en los centros educativos, convirtiéndose en figuras interdependientes e igualmente necesarias para lograr el éxito en este objetivo. 


\title{
Special educational needs: a conceptual burden for inclusive education in Spain
}

\begin{abstract}
In Spain, teachers currently receive little training on attention to diversity, and qualification of Education lacks the specialty which before was preparing school counselors, whose work is essential to the process of educational inclusion. Furthermore, the concept of special educational needs (SEN) seems to be assuming a burden for both the students themselves who it refers to, and the advances made to try to attend to diversity. In order to know the opinion of the educational sector regarding these two issues, a survey was conducted to 160 professionals. The results showed that the participants consider the role of the school guidance a key in this process, and that they present a reductionist view of diversity. Therefore, the training for both teachers and counselors should be strengthened in order to face this problem and the concept of SEN should be modified to avoid reductionism on diversity.
\end{abstract}

Keywords: Inclusive education. Special educational needs. Special education. School guidance.

\section{Necessidades educativas especiais: um lastro conceitual para a educação inclusiva na Espanha}

\section{Resumo}

Atualmente em Espanha, os professores recebem parca formação em atenção à diversidade e a licenciatura em Educação não tem a especialização que antes preparava os orientadores pedagógicos e cujo trabalho é essencial para o processo de inclusão educacional. Além disso, o conceito de Necessidades Educativas Especiais (NEE) parece ter-se tornado um obstáculo, tanto para os alunos, aos quais faz referência, como para os progressos que se têm vindo a fazer para responder à questão da diversidade. Com o objetivo de se conhecer a opinião do sector da educação a respeito destas duas questões, foi realizada uma pesquisa com 160 profissionais da área no ativo. Os resultados mostraram que os inquiridos consideram o papel dos orientadores pedagógicos um ponto-chave neste processo, mas detêm uma perspetiva redutora sobre a diversidade. Por conseguinte, a formação de professores e orientadores deveria ser reforçada no intuito de lidar com esta problemática e o conceito de NEE deveria ser alterado para precaver reducionismos.

Palavras-chave: Educação inclusiva. Necessidades especiais. Ensino especial. Orientação educacional. 


\section{Referencias}

AINSCOW, M. Tendiéndoles la mano a todos los estudiantes: algunos retos y oportunidades. Siglo Cero, v. 30, n. 1, p. 37-48, ene.-mar. 1999.

AMERICAN PSYCHIATRIC ASSOCIATION - APA. Diagnostic and statistical manual of mental disorders: DSM-IV-TR: text revision. 4. ed. rev. Washington: American Psychiatric Press, 2000.

ARNÁIZ, P. Educación inclusiva: una escuela para todos. Málaga: Aljibe, 2003.

BOOTH, T.; AINSCOW, M.; KINGSTON, D. Index para la inclusión: desarrollo del juego, el aprendizaje y la participación en educación infantil. Bristol: CSIE, 2006.

DUK H., C.; LOREN G., C. Flexibilización del currículum para atender la diversidad. Revista Latinoamericana de Educación Inclusiva, v. 4, n. 1, p. 187-210, abr. 2010.

DURÁN GISBERT, D.; GINÉ GINÉ, C. La formación del profesorado para la educación inclusiva: un proceso de desarrollo profesional y de mejora de los centros para atender la diversidad. Revista Latinoamericana de Educación Inclusiva, v. 5, n. 2, p. 153-170, oct. 2011.

DYSON, A. Dilemas, contradicciones y variedades de la inclusión. In: Verdugo, M.; Jordán de Urríes, F. (Eds.). Apoyos, autodeterminación y calidad de vida. Salamanca: Amarú, 2001. p. 145-60.

ECHEÍTA, G. Educación para la inclusión o educación sin exclusiones. Madrid: Narcea, 2007.

ESPAÑA. Ley Orgánica 1/1990, de 3 de octubre, de Ordenación General del Sistema Educativo. Boletín Oficial del Estado, 4 oct. 1990.

. Ley Orgánica 2/2006, de 3 de mayo, de Educación (LOE). Boletín Oficial del Estado, 4 mayo 2006.

. Ley Orgánica 8/2013, de 9 de diciembre, para la Mejora de la Calidad Educativa (LOMCE). Boletín Oficial del Estado, 10 dic. 2013.

. Ley Orgánica 9/1995, de 20 de noviembre, de la participación, la evaluación y el gobierno de los centros docentes (LOPEG). Boletín Oficial del Estado, 21 nov. 1995. 
GARCÍA-BARRERA, A. Las necesidades educativas personales: un concepto nuclear latente en educación. 2013.373 f. Tesis - Facultad de Formación del Profesorado, Universidad Autónoma de Madrid, Madrid, 2013.

GARCÍA PASTOR, C. Inclusión: una forma comprometida de trabajar en la escuela. In: Rondal, J. Pérez, J. y Nadel, L. Revisión de los últimos conocimientos. Madrid: Espasa, 2000. p. 123-40.

GRAU RUBIO, C. La formación de profesores de educación especial. [S.I.], 2001. Disponible en: $<$ http://redes-cepalcala.org/inspector/ DOCUMENTOS\%20Y\%20LIBROS/FORMACION/FORMACION\%20 DE\%20PROFESORES\%20DE\%20EE.doc>. Acceso en: 10 feb. 2014.

INSTITUTO DE TECNOLOGÍAS EDUCATIVAS - ITE. Educación inclusiva: iguales en la diversidad. Madrid: Ministerio de Educación, 2009.

LEÓN GUERRERO, M. J. La formación del profesorado para una escuela para todos: un análisis de los planes de estudio del maestro especialista en Educación Primaria y en Educación Especial de las universidades españolas. Profesorado, v. 3, n. 2, p. 11-38, abr. 1999.

LÓPEZ, M.; ECHEÍTA, G.; MARTÍN, E. Dilemas en los procesos de inclusión: explorando instrumentos para una comprensión de las concepciones educativas del profesorado. Revista Latinoamericana de Educación Inclusiva, v. 4 , n. 2, p. 155-76, oct. 2010.

LÓPEZ TORRIJO, M. La inclusión educativa de los alumnos con discapacidades graves y permanentes en la Unión Europea. Relieve, v. 15, n. 1, p. 1-20, ene..-jun. 2009.

MARTÍNEZ-GARRIDO, C.; KRICHESKY, G. J.; GARCÍA-BARRERA, A. El orientador escolar como agente interno de cambio. Revista Iberoamericana de Educación, v. 54, p. 107-22, sep.-dic. 2010.

MASLOW, A. H. A theory of human motivation. Psychological Review, v. 50, p. 370-96, ene. 1943.

NORWICH, B. Dilemmas of difference, inclusion and disability: international perspective placement. European Journal of Special Needs Education, v. 23, n. 4, p. 287-304, oct..-dic. 2008.

ORGANIZACIÓN MUNDIAL DE LA SALUD - OMS. CIE-10: trastornos mentales y del comportamiento. Madrid: Meditor, 1992. 
PALOMARES RUÍZ, A. Un profesorado para atender a la diversidad. Ensayos, v. 13, p. 283-294, ene. 1998.

PARRILLA, A. La voz de la experiencia: la colaboración como estrategia de inclusión. Aula de Innovación Educativa, v. 121, p. 43-8, ene. 2003.

PUJOLÁS, P. Atención a la diversidad y aprendizaje cooperativo en la educación obligatoria. Málaga: Aljibe, 2001.

REYNOLDS, M.C. An historical perspective: the delivery of special education to midly disabled and at-risk students. Remedial and Special Education, v. 10, n. 6, p. 7-11, nov..-dic. 1989. http://doi.org/10.1177/074193258901000604

UNESCO. Una educación de calidad para todos los jóvenes: reflexiones y contribuciones In: CONFERENCIA INTERNACIONAL DE EDUCACIÓN DE LA UNESCO, 47., 2004, Ginebra, 2004. Reflexiones y contribuiciones... Ginebra: Unesco, 2005.

WARNOCK, M. Special educational needs: report of the Committee of Enquiry into the Ecucation of Handicapped Children and Young People. London: Her Majesty's Stationary Office, 1978.

WOLSFENSBERGER, W. (1994). Let's hang up "quality of life" as a hopeless term. In: GOODE, D. (Ed.). Quality of life for persons with disabilities: international perspectives and issues. Cambridge: Brookline, 1994. p. 285-321.

\section{Informações da autora}

Alba García-Barrera: Doctora en Educación. Profesora del área de Educación en Universidad a Distancia de Madrid - UDIMA. Contato: alba.garcia@udima.es 